\title{
The Study of Multi-Expression Classification Algorithm Based on Adaboost and Mutual Independent Feature
}

\section{Liying Lang, Zuntao Hu}

Department of Electrical and Information, Hebei University of Engineering, Handan, China.

Email: huzuntao@126.com

Received June 20 ${ }^{\text {th }}, 2011$; revised August $13^{\text {th }}, 2011$; accepted August 22 ${ }^{\text {nd }}, 2011$.

\begin{abstract}
In the paper conventional Adaboost algorithm is improved and local features of face such as eyes and mouth are separated as mutual independent elements for facial feature extraction and classification. The multi-expression classification algorithm which is based on Adaboost and mutual independent feature is proposed. In order to effectively and quickly train threshold values of weak classifiers of features, Sample of training is carried out simple improvement. We obtain a good classification results through experiments.
\end{abstract}

Keywords: Adaboost Multi-Expression Classification Algorithm, Local Feature, Feature Extraction, Sample Training

\section{Introduction}

Due to increasingly wide application, Facial multi-expression classification which is widely concerned by governments and research institutes is becoming a challenging research topic in field of pattern recognition. Currently there are many main methods used for facial expression classification, such as Euclidean distance, Support vector machine (SVM), Neural network(NN), Hidden Markov(HMM), Adaboost and Linear discriminant analysis (LDA), etc. Adaboost algorithm which owns higher speed and higher detection rate has been successfully applied in the field of face detection. Most of facial expression changes exist in eyes and mouth, so features of eyes and mouth are treated as mutual independent elements [1]. The method can greatly reduce redundancy and improve the speed of training threshold values. However, if we want to use the algorithm to facial multi-expression classification, there is a main problem. When error recognition rate of weak classifiers of Adaboost is higher, overall recognition rate of the algorithm will reduce to zero exponentially, key problem is to train a weak classifier threshold value accurately and fast. So we improve training samples, and negative samples is proposed in this paper [2]. Threshold value of weak classifier is the most crucial part. Positive samples contain only images of eyes or mouth, while negative samples are removed eyes and mouth. So the multi-expression classification algorithm which is based on Adaboost and mutual independent feature is proposed. Experimental results prove that false recognition rate is almost close to zero.

\section{Adaboost Multi-Expression Classification Algorithm}

Basic idea of Adaboost algorithm is to use a large number of weak classifiers to add up together to form a very strong classification through a certain method. In the algorithm, each training sample is assigned a weight, and it demonstrates a probability of some weak classifiers which can be selected into training set. If a sample is accurately classified by the current weak classifier, its weight will be reduced. On the contrary, if a sample is not properly classified, the weight is to be raised accordingly. In this way, Adaboost algorithm can focus on more difficult samples [2,3].

A weak classifier $H_{j}(x)$ consists of the following three parts: rectangle feature value $f_{j}(x)$, a classification threshold value $\theta_{j}$ and a direction sign $p_{j}\left(p_{j}=\right.$ $0,1)$. They are in line with the following relationship [4]:

$$
H_{j}(x)=\left\{\begin{array}{l}
j, p_{j} f_{j}(x)<p_{j} \theta_{j}, j=1,2,3,4,5,6,7 \\
0, \text { else }
\end{array}\right.
$$

We obtain the minimum threshold value $\theta_{\min (f i)}$ and the maximum threshold value $\theta_{\max (f i)}$ of eyes and mouth, making $\theta_{\min (f i)}<\theta_{j}<\theta_{\max (f i)} . H_{j}(x)$ is one of seven 
facial expressions .Positive samples of human eyes and mouth contain a variety of gestures, such as eyes open or closed, wearing glasses, mouth open or closed, etc. Negative samples do not contain any of eyes and mouth. The algorithm is showed as follows:

The first step:

Giving samples $\left\{\left(x_{1}, y_{1}\right),\left(x_{2}, y_{2}\right), \cdots\left(x_{n}, y_{n}\right)\right\}, x_{i}$ is input training sample, $y_{i} \in\{0,1\}$ indicates positive sample or negative sample, $\mathrm{L}$ is the number of positive samples , $\mathrm{m}$ is the number of negative samples. $\mathrm{T}$ is the number of iterations of strong classifier. $D_{t}$ is the probability distribution of sample weigh, and making

$D_{1}(i, l)=1 / 7 n, i=1, \cdots, n, l=1, \cdots, 7$.

$n$ is the total number of samples, $\mathrm{j}$ is one of seven types of expression [5]

$$
y_{i}[l]=\left\{\frac{+1, i \in y}{-1, i \notin y}\right.
$$

The second step:

1) We train a weak classifier of each feature of sample $x_{i}$, whose output is multi-class.

2) In weight distribution $D_{t}$, we select the best classifier $h_{t}$ from various weak classifiers, making the classification error rate minimum. The following formula obtaining the maximum:

$$
\begin{gathered}
r_{t}^{\prime}=\sum_{i, l} D_{t}(i, l) y_{i}[l] h_{t}\left(x_{i}, l\right) \\
r_{t}=\max \left(r_{t}^{\prime}\right)=\max \sum_{i, l} D_{t}(i, l) y_{i}[l] h_{t}\left(x_{i}, l\right) \\
h_{t}(x)=h_{t}\left(x_{i}, l\right)
\end{gathered}
$$

3) According to the classification performance, the weight of classifier will be changed.

$$
a_{t}=\frac{1}{2} \ln \left(\frac{1+r_{t}}{1-r_{t}}\right)
$$

4) Sample weights will be updated and normalized.

$$
D_{t+1}(i, l)=\frac{D_{t}(i, l) \exp \left(-a_{t} y_{i}[l] h_{i}(i, l)\right)}{z_{t}}
$$

$z_{t}$ is normalization constant

5) For the normalization constant $z_{t}$, weight distribution $D_{t+1}$ is made a probability density.

$$
z_{t}=\sum_{i, l} D_{t}(i, l) \exp \left(-a_{t} y_{i}[l] h_{t}\left(x_{i}, l\right)\right)
$$

The third step:

The final strong classifier can be obtained as follows:

$$
\frac{\left|\left\{i: H\left(x_{i}\right) \neq y_{i}\right\}\right|}{n} \leq \sqrt{c-1} \prod_{t=1}^{T} z_{t}
$$

The algorithm error rate fits the following inequality on the training set at this time $[4,6]$.

$$
H(x, l)=\operatorname{sign}\left(\sum_{t=1}^{T} a_{t} h_{t}(x, l)\right)
$$

\section{Training and Testing of Local Features}

\subsection{Training}

We need a large number of samples in sample training, which is an important characteristic about Adaboost algorithm. Selection of sample is very important, which determines the effect of the classification. We divide samples into positive and negative. Eyes and mouth are treated as mutual independent feature elements. In order to easily obtain the weak classifiers threshold values, we train the eyes and mouth respectively. When we get the minimum threshold value or the maximum threshold value of kinds of expression, chenges of eyes and mouth will lead to huge variations. So classifiers are very difficult to get threshold values accurately and effectively. Therefore, specific training process is divided into four steps [7]. The first step, we use the positive samples to train, obtaining the threshold values quickly. The second step, in order to adjustment the threshold values appropriately, we use whole face images to train. As the false detection always occurs in eye or mouth, we use eye images and mouth images to further adjust the threshold parameters in third step. The fourth step is to use the new negative sample proposed in the paper to reduce the false detection rate.

\subsection{Detection}

The specific detection process is as follows: when there is a image to detecte, we use different rectangular boxes, whose sizes are generally from small to large, to scan the whole image. The size of the smallest rectangle box is normalized, For example $24 \times 24$ pixel. Each rectangular box move a pixel from right to left until it reaches edge of the image. When rectangular box scans the whole image at this level, rectangular box enlarge a certain pixel to next scanning. Therefore the largest rectangle box is several times larger than the smallest rectangular box. During the scan, each rectangular area can be carried out classification decision. The purpose of scan is to find a specific facial feature region. Therefore, if detection area can be adopted by the classification, it proves to find a person's eyes or mouth, else detection will be stopped. If any of rectangular area can not be adopted by the classifier, the region does not exist any eyes or mouth. Of course, when dimensions of the rectangular area enlarge in proportion, threshold parameters of weak classifier also amplified by the same proportion. 


\section{Experimental Results and Analysis}

\subsection{Facial Expression Image Database}

We selected Japanese women expression database of the Kyushu University JAFFE and Our self-built facial expression database in experiments. The JAFFE database is made up 213 individual images of ten persons, and each person shows anger, disgust, fear, happiness, sadness, surprise and neutral. There are 2 - 4 images for every face expression, and images are all $256 \times 256$ grayscale images. Our self-built database composes of seven individuals, each person also shows seven basic facial expressions, and pictures are also $128 \times 128$ grayscale images $[3,8]$. As images obtain from two different databases, there are very huge difference. To be able to quickly and accurately classify the facial expression, images need appropriate pretreatment. In this paper, we use geometric normalization and the intensity normalized to improve the image quality. We get the mouth images, the eye images and the whole face images. Figure 1 shows the images selected from the JAFFE database and self-built database. Figure 2 shows the pretreatment image and the negative sample.

\subsection{Experimental Results}

We selected 120 facial expression images of six persons from JAFFE database and selected 42 images of three persons from self-built database in experiments. Table 1 shows different experimental results between JAFFE database and self-built database. Figure 3 shows the recognition rates of the seven different facial expressions $(1$ is on behalf of happiness, 2 is neutral, 3 is sadness, 4 is surprise, 5 is disgust, 6 is angry, 7 is fear). It is can be seen from the Table 1 that recognition rates in JAFFE are much more higher than the self-built database. The reason is that the facial expression in self-built database is not exaggerated, resulting in a huge difference between the experimental results.

\subsection{Experimental Analysis}

In experiments, the number of iteration is set 60 . With the number of iterations changing, the error recognition rate will be very different. We compared two different expressions in a group, the experimental results show that recognition rate of happiness and surprise are more higher than disgust, sadness and neutral. The reasons are that facial expression changes of happiness or surprise is much more obvious, feature extraction is more easier, and classification get a small error. What's more, it can be seen from Figure 4 that the error recognition rate of the first 30 iterations reduces more faster than the 30 times later. Especially you can see that when the iteration is to 50 , the change of error rate is very small. According

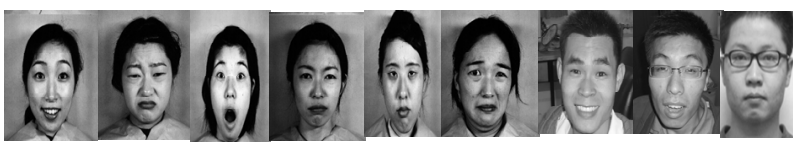

Figure 1. Parts of samples from JAFFE and our self-built database.

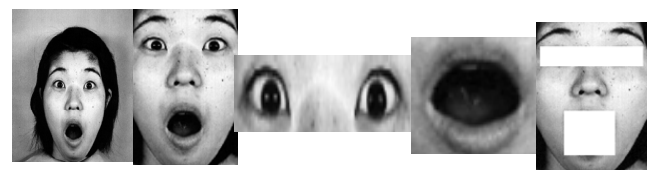

Figure 2. The pretreatment image and the negative sample.

Table 1. The detection results of the method.

\begin{tabular}{cccc}
\hline Database & $\begin{array}{c}\text { Total } \\
\text { sample number }\end{array}$ & $\begin{array}{c}\text { Wrong detection } \\
\text { number }\end{array}$ & $\begin{array}{c}\text { Correct } \\
\text { detection rate }\end{array}$ \\
\hline $\begin{array}{c}\text { JAFFE } \\
\text { Self-built } \\
\text { database }\end{array}$ & 120 & 13 & $89.16 \%$ \\
\hline
\end{tabular}

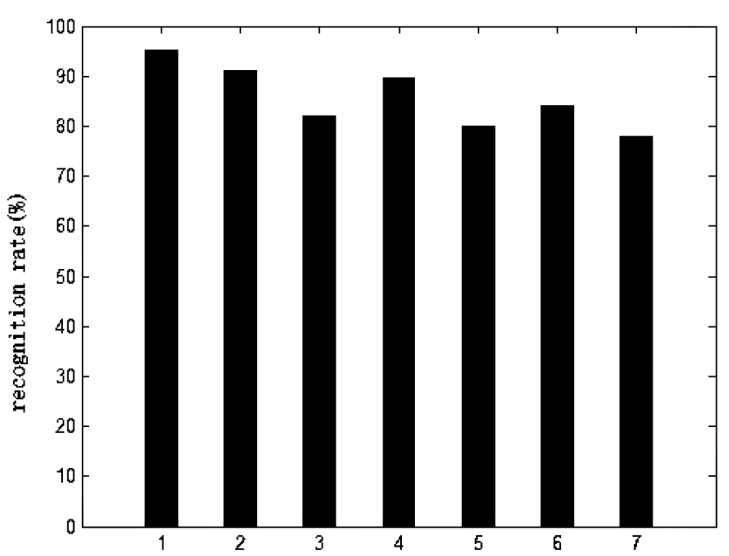

Figure 3. The results of seven types of expression recognition rate.

to the characteristics of this change, without affecting the recognition rate, we can reduce the number of iterations to accelerate the classification speed.

\section{Conclusions}

In the paper, Adaboost algorithm, which has been successfully applied in the field of face detection, applies to the facial expression classification. We introduce the basic principle of Adaboost milti-expresion classification algorithm and demonstrate the process of training and testing in detail. Because changes of facial expression mainly exist in eyes and mouth, we treat eyes and mouth as mutual independent elements, which improved the speed of training threshold value. The negative samples is proposed and used in training and testing. The experimental results demonstrated the feasibility of the method, which obtained a good recognition result. 

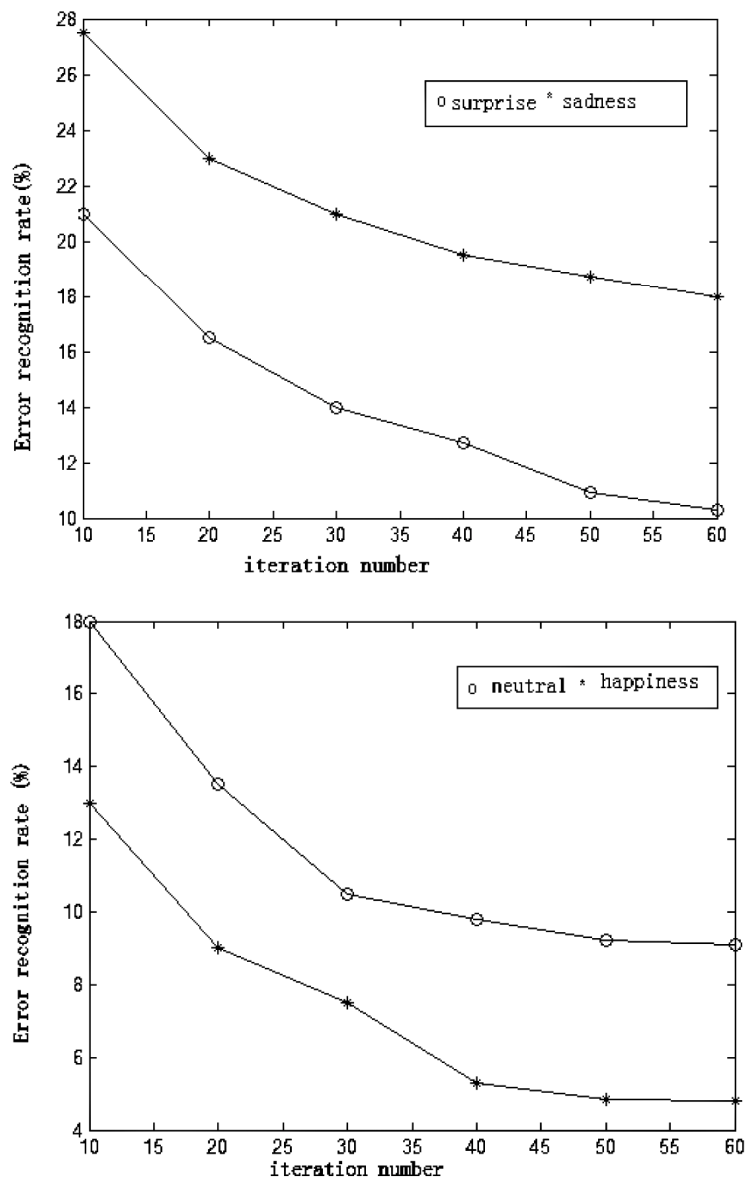

Figure 4. Error recognition rate changing with the iteration number.

\section{Acknowledgements}

This work is supported by Department of Electrical and Information, Government of Handan, China. Authors are thanking to them for their sponsorship to do this work.

\section{REFERENCES}

[1] X. Z. Zhao and X. F. Zhu, "Adaboost Algorithm Based on Detection of the Human Eye of Sample Selection," Computer Technology and Development, Vol. 20, No. 2, 2010, pp. 12-16.

[2] H. Y. Wang and X. Li, "Facial Expression Recognition Based on Improved Gabor Transformation and Adaboost Algorithm," Journal of Liaoning University of Technology (Natural Science Edition), Vol. 30, No. 1, 2010.

[3] M. Qiao and Y. J. Chen, "Feature Extraction Methods on Facial Expression Recognition,” Journal of Chongqing Institute of Technology, Vol. 22, No. 6, 2008, pp. 118122.

[4] G. L. Yang, Z. L. Wang and J. X. Ren, "Adaboost Algorithm Is Used for Facial Expression Recognition,” Computer Engineering, Vol. 25, No. 4, 2005, pp. 78-83.

[5] Y. Ma, X. Q. Ding and Z. E. Wang, "Robust Preciseeye Location under Probabilistic Framework," Republic of Korea, Seoul, 2004, pp. 339-344.

[6] J. Zhao and L. A. Xu, "Target Detection Based on Adaboost Algorithm,” Computer Engineering, Vol. 30, No. 2, 2004, pp. 78-83.

[7] Y. Huang and Z. L. Ying, "Classifier Combination Based Expression Recognition,” Computer Engineering and Applications, Vol. 43, No. 25, 2007.

[8] P. S. Aleksic and A. K. Katsaggelos, "Automatic Facial Expression Recognition Using Facial Animation Parameters and Muhistream HMMs," IEEE Transactions on Information Forensics and Security, Vol. 1, No. 1, 2006, pp. 3-11. doi:10.1109/TIFS.2005.863510 